\title{
Serological diagnosis of Bacteroides fragilis infections by a complement fixation test
}

\author{
CONSTANCE A. C. ROSS AND R. F. GILMORE
}

From the Microbiology Laboratory, Ayrshire Central Hospital, Irvine, KA12 8SS, UK

SUMMARY Paired specimens of serum from patients from whom Bacteroides fragilis had been isolated were tested by complement fixation against a crude $B$. fragilis antigen. A high titre or a rise in titre to $B$. fragilis was obtained in each of five patients with infection after abdominal surgery but in none of 11 patients with postpartum pyrexia nor in nine with vaginitis.

Within recent years it has been increasingly recognised that endogenous infection with the bacteroides group of organisms, mainly Bacteroides fragilis, may follow gastrointestinal or pelvic surgery (Gillespie and Guy, 1956; Ledger et al., 1971). Thus, isolation of $B$. fragilis from specimens of pus from patients with postoperative infections is presumptive evidence that this is the causative organism. Isolation of $\boldsymbol{B}$. fragilis from the vagina of patients with genital tract infections is much more difficult to interpret since this species is a normal inhabitant of the vagina.

The purpose of the present study was to find out if the demonstration of a serum antibody response to $B$. fragilis might help to determine the pathogenic significance of the isolation of this species. For antibody tests we used the complement fixation $(C F)$ technique since it is widely used in our laboratory for serodiagnosis of bacterial and viral infections.

\section{Material and methods}

IDENTIFICATION OF BACTEROIDES

Isolates were identified as Bacteroides sp on the basis of cultural and morphological appearances (anaerobic Gram-negative rods): further identinication as $\boldsymbol{B}$. fragilis, the commonest Bacteroides sp associated with clinical infections, was made if the isolate showed the characteristic antibiogram (Sutter and Finegold, 1971).

PREPARATION OF BACTEROIDES CF ANTIGEN For preparation of $\mathrm{CF}$ antigen a strain of $B$. fragilis isolated from a post-hysterectomy wound infection was subcultured on six blood agar plates so as to give a confluent growth when incubated for 48 hours

Received for publication 8 May 1978 anaerobically. The growth was washed off each plate with $4 \mathrm{ml}$ veronal buffer (Grist et al., 1974). The pooled washings were frozen to $-20^{\circ} \mathrm{C}$ and thawed twice; the whole suspension constituted the antigen; it was stored at $-20^{\circ} \mathrm{C}$. The optimum dilution of antigen for CF was determined by means of a microtechnique in a chessboard titration (Grist et al., 1974) against serum from a patient recovering from $B$. fragilis septicaemia. This serum was used as the positive control throughout our tests.

PATIENTS AND TEST SERA

Paired specimens of serum, having an interval of 10-21 days between members of each pair, were requested from all patients in three groups from whom $B$. fragilis was isolated in our laboratory during the year March 1975 to March 1976. Group 1 comprised infections after abdominal surgery; group 2 women with postpartum pyrexia; and group 3 women suffering from vaginitis. From high vaginal swabs from women in groups 2 and 3 a heavy growth of $\boldsymbol{B}$. fragilis had been obtained in anaerobic cultures. Paired specimens of serum were received as follows: from five in group 1, from 11 in group 2, and from nine in group 3. Specimens from each patient were tested in parallel against the optimum dilution of the antigen.

\section{Results}

An antibody response was not detected (CF titres $<8$ ) in serum from any of the 11 women with postpartum pyrexia nor from the nine women with vaginitis. In contrast, an antibody response to $B$. fragilis was found in four patients with infections after abdominal surgery, from whose wounds the organism was isolated, and in a fifth patient with a 
Table Postoperative B. fragilis Infections

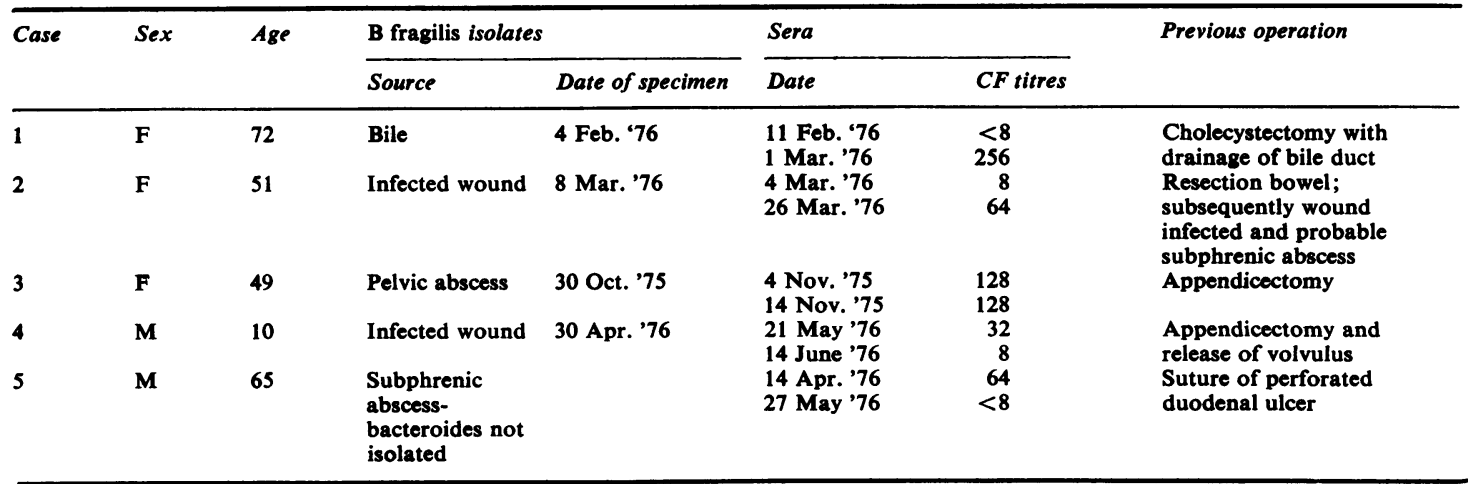

subphrenic abscess but from whom Bacteroides sp was not isolated (Table). There were two patients with a rise in titre (cases 1 and 2), one with a high static titre (case 3), and two with a fall in titre (cases 4 and 5).

The convalescent serum from case 1 , which gave the highest titre, namely, 1 in 256, was tested also in chessboard titrations against antigens prepared from other strains of $\boldsymbol{B}$. fragilis isolated from four patients with the following clinical infections: vaginitis, skin ulcer, penile sore, and otitis media. The same serum titre was obtained with all antigens.

\section{Discussion}

At the time of this study identification of $B$. fragilis was relatively imprecise, and we did not attempt to identify subspecies as advocated by later workers (cited by Willis, 1977). Thus we do not have any evidence that the $C F$ antigen related to our study was common to all species and subspecies of $B$. fragilis. However, the significant $\mathrm{CF}$ antibody response to the antigen in the five patients with infections after abdominal surgery suggests that there may be a common CF antigen for all species of $B$. fragilis or for all strains of $\boldsymbol{B}$. fragilis fragilis, which is the subspecies most often implicated in clinical infections (Willis, 1977). It may be relevant that immunochemical studies have shown that $B$. fragilis fragilis has a distinct outer membrane, which contains a protein component seemingly shared by many strains of this subspecies (Kasper and Seiler, 1975).

It appears from our findings that demonstration of a rise in CF titre in paired specimens of serum, (or a high CF titre when suitably paired specimens are not available) in relation to a crude $B$. fragilis antigen may help in assessing the aetiological significance of an isolation of the organism or indicate infection in patients with suspected bacter- oides infection from whom the organism has not been isolated. Further work is required in order to establish the specificity of the reaction not only within the $B$. fragilis species but also in respect of other species of bacteroides.

Our failure to detect any antibody responses in the patients with vaginitis or postpartum pyrexia suggests that the strains of $\boldsymbol{B}$. fragilis isolated were commensals in these patients, or were antigenically unrelated to the strain from which the $C F$ antigen was prepared. It may be, however, that in vaginal in? fections bacteroides is only locally invasive and does not elicit an antibody response.

We thank Mr J. Moore, Mr A. Murison, and Dr D. Smith for clinical cooperation.

\section{References}

Gillespie, W. A., and Guy, J. (1956). Bacteroides in intra-abdominal sepsis. Lancet, 1, 1039-1042.

Grist, N. R. G., Ross, C. A. C., and Bell, E. J. (1974). Diagnostic Methods in Clinical Virology, 2nd edition, pp. 81-89. Blackwell, Oxford.

Kasper, D. L., and Seiler, M. W. (1975). Immunochemical characterization of the outer membrane complex of Bacteroides fragilis subspecies fragilis. Journal of Infectious Diseases, 132, 440-450.

Ledger, W. J., Sweet, R. L., and Headington, J. T. (1971). Bacteroides species as a cause of severe infections in obstetric and gynaecologic patients. Surgery Gynecology and Obstetrics, 133, 837-842.

Sutter, V. L., and Finegold, S. M. (1971). Antibiotic disc susceptibility tests for rapid presumptive identification of Gram-negative anaerobic bacilli. Applied Microbiology, 21, 13-20.

Willis, A. T. (1977). Anaerobic Bacteriology in Clinical and Laboratory Practice, 3rd edition, Chapter 5, Butterworths, London.

Requests for reprints to: $\mathrm{Dr}$ Constance A. C. Ross, Microbiology Laboratory, Ayrshire Central Hospital, Irvine, Ayrshire, KA12 8SS. 